\title{
COGNITIVE LINGUISTICS
}

\section{Function of a Lawyer: Frame Approach}

\section{O. V. Dunaievska}

Taras Shevchenko National University of Kyiv, Ukraine

Corresponding author. E-mail: o.dunayevska@gmail.com

Paper received 02.02.20; Accepted for publication 18.02.20.

\section{https://doi.org/10.31174/SEND-HS2020-223VIII37-08}

\begin{abstract}
The paper addresses the issue of the application of a frame structure in teaching Legal English as a Foreign Language (LEFL). The function of a lawyer is derived through frame analysis of "Lawsuit" stereotypical situation. A frame is determined to be the cognitive area representing the knowledge on particular situation, and action frame model is utilized primarily as the basic medium to refer to "Lawsuit" situation in terms of its "participants", their "actions". Secondly, the implicit slot of the frame Lawsuit "instruments" localizes the lexis representing those participants of Lawsuit situation where a lawyer belongs to, making a lawyer functions as an instrument in "Lawsuit" situation.
\end{abstract}

Keywords: action frame, evoked frame, invoked framing, lawsuit, lawyer, Teaching Legal English as a Foreign Language.

Modern science is crossing boundaries between disciplines by snowballing the new opportunities for interdisciplinary studies which share one thing. The latter is a human: their spiritual, cognitive, physical, social, and other aspects of existence. Lately, more and more talks arise in sphere of successful human activity that makes the latter be one of the most vulnerable issues ever discussed. Though a global workforce market is strongly affected by considerable flow of higher establishments' graduates, there is quite a critical situation with properly educated specialists. The paper addresses the issue combining professional communicative performance of a future legal specialist and its elaboration through modifying approaches to teaching Legal English as Foreign Language (LEFL). The processes mentioned are reflected through adaptation to artificial environment of a conventional university's Legal English class.

A quite large number of researches touching upon the language factor in legal environment can be mentioned as a considerable contribution was made by terminologists, applied linguists, specialists in pedagogy, philosophy, and other sciences. Particularly, an interdisciplinary research of law, its associated texts and norms of legal discourse were awarded specific attention from prospective of language philosophy, its social impact involving the elements of critical semiotics and rhetoric by P. Goodrich [6]. The comprehensive study of legal English as a communication medium is introduced by $\mathrm{P}$. Tiersma [10], L. M. Solan, J. Ainsworth, and R. W. Shuy [9]. Thus, some scope is still left for the research combining practical teaching methods with semasiological and onomasiological approaches. The paper aims to outline the perception of a lawyer as a representative of a legal profession based on cognitive and semantic modeling of a legal process environment or a lawsuit with its practical application for the learning purposes. It is hypothesized that frame semantics serves as a binding instrument between theory of linguistic methods and their utilization in LEFL teaching.

The objectives of the research are:

- to determine the universal tool for representation of knowledge on particular situation;
- to model a stereotypical situation of legal environment;

- to outline the role of a lawyer in stereotypical situation;

- to localize the slot of the frame which is responsible for perceiving and understanding of a lawyer's function;

- to determine the way of introduction of the study's results to the LEFL class.

The fact that people encounter the situation relying upon its frame, the already stored in the memory and a preserved fragment of person's cognitive worldview was extensively explored in 1987 by M. [8]. The scientist highlighted the idea in "A Framework for Representing Knowledge," where the notion of a Frame was introduced in relation to stereotyped situation as datastructure of the latter. The idea of a Frame was alternative to already used notions of "scheme" [1] and "paradigm" [7]. According to Fillmore Ch.J. these are communicative units, which translate the knowledge on particular situation or event [4, p. 118]. Obviously, the means of verbal and non-verbal communication that fill the definite parts of the frame (slots) with appropriate words give an excess to its superior cognitive structure [5, p. 223] - concept. The encountering a familiar situation launches the process of evoking. Due to non-finite nature a frame it is capable of evoking the limitless number of its parts embedded in cognitive worldview of an individual pertaining to a definite situation or an event [4, p. 130]. The cases where there are no records on encountered events in the cognitive worldview of an individual the invoking process or accumulation of knowledge starts and, thus, the event is served by an invoked frame. Such information "gaps" or "blank spaces" are supplied with the information from universal knowledge database coming from the general experience of a society or comprehended directly from the text or discourse [4, p. 20]. The collection of frames create frame-systems [8] or frame-nets [10]. In other words, familiar events evoke frames relevant to the situation and unfamiliar ones add new data (based on experience which is being earned) attaching them to already exiting frame-net by invoked framing. Each frame is actualised through particular number of terminals or "slots" [8] 
containing the various data. The latter are coded by verbal means the study of which will hypothetically give an access to the hole structure. Pertaining to the learning environment of teaching LEFL classes the basic frame the learners encounter is the frame LAWYER. The importance lies in what LEFL class learner is ready to evoke concerning the notion of their future legal profession that reflects the basis of understanding of frame LAWYER firstly, and the prospects of connecting the latter with the more extended network secondly. As a matter of fact, LEFL class is based on invoking the network of frames connected to super-frame LEGAL PROFESSION. The lexical units used in it are spread among the slots of the frames and sub-frames of the previously mentioned super-frame. One of the basic associations which are connected with a legal profession is a lawsuit, since the latter is the basic case the lawyer is engaged in (referring to phases of preparing for a lawsuit, participating in different forms of alternative dispute resolution or in the court hearing, appealing or enforcement). Moreover, the frame study extended by a semantic research of a lawsuit notion will shed the light on the mental perception of a lawyer's function.

Owing to the fact that the dictionaries do not offer the same definition of a lawsuit, one can be constructed as a collective description. According to online dictionaries: The American Heritage Dictionaryi, Collins Dictionary ${ }^{\mathrm{ii}}$, Macmillan Dictionary ${ }^{\mathrm{iii}}$, Cambridge Dictionary ${ }^{\text {iv }}$, Dictionary Law.com ${ }^{v}$, Webster's New World Law Dictionary $^{\mathrm{vi}}$, The Electronic Law Library and Glossary of Legal terms ${ }^{\text {vii }}$, Business Dictionary viii the notion of a lawsuit gets verbalized in the following way:

- to the name of the process belong lexical units: proceeding $\times 3$, action $\times 4$, claim $\times 1$, dispute $\times 3$, case $\times$ 4 , causa $\times 1$, problem $\times 1$, act $\times 1$, suit $\times 2$;

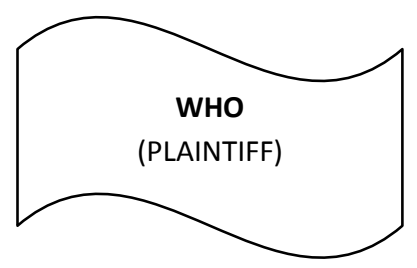

The slots of the schematized action frame (PLAINTIFF, TO ACT, DEFENDANT) are filled with the diverse lexical material, depending on the field of law the frame serves. Therefore action frame model is a universal container for preserving information connected with any field of law. It is necessary to mention that Fig. 1 depicts only explicit slots of action frame LAWSUIT and thus the situation of a lawsuit explicates only two participants (a plaintiff, a defendant) and the actions that constitute a core of the frame. The periphery
- to the characteristic localization described by the notion refer: court of law $\times 9$, civil court $\times 1$;

- the participants of the situation are described by units: two parties $\times 2$; two people $\times 2$; individuals/organizations $\times 2$; person or entity $\times 1$; plaintiff $\times$ 1; defendant $\times 1$;

- to the actions mentioned belong: to bring/start $\times 3$, to seek a legal remedy $\times 2$, to act against (start an action against) $\times 3$, to obtain $\times 1$, to sue $\times 1$.

The analysis of 12 definitions offered by various online dictionaries clearly states the absence of a conventional understanding in terms of variability of the lexis used definition of a lawsuit. Therefore, the collective definition aims to unite the most commonly used lexical units, that characterize a lawsuit as: an action or a case where two parties or organizations act against each other within the court of law. Based on the previous it is outlined that the key characteristic for a frame LAWSUIT is dynamics driven by actions of two parties in the situation of a lawsuit. The type of a structure serving the situation of a lawsuit can be described as a surface semantic frame, which involves "actioncentered meanings of words, qualifiers and relations concerning participants, instruments, trajectories and strategies, goals, consequences and side-effects" [8] or an action frame, concentrating the actions of someone towards someone else: WHO --- ACTS on --- WHO [Zabotynska 2010].

A frame LAWSUIT reflects a legal event /stereotypical situation or a process with distinct implicit direction: from a plaintiff to a defendant. The latter crystallizes its opportunity to develop through the following action frame scheme:

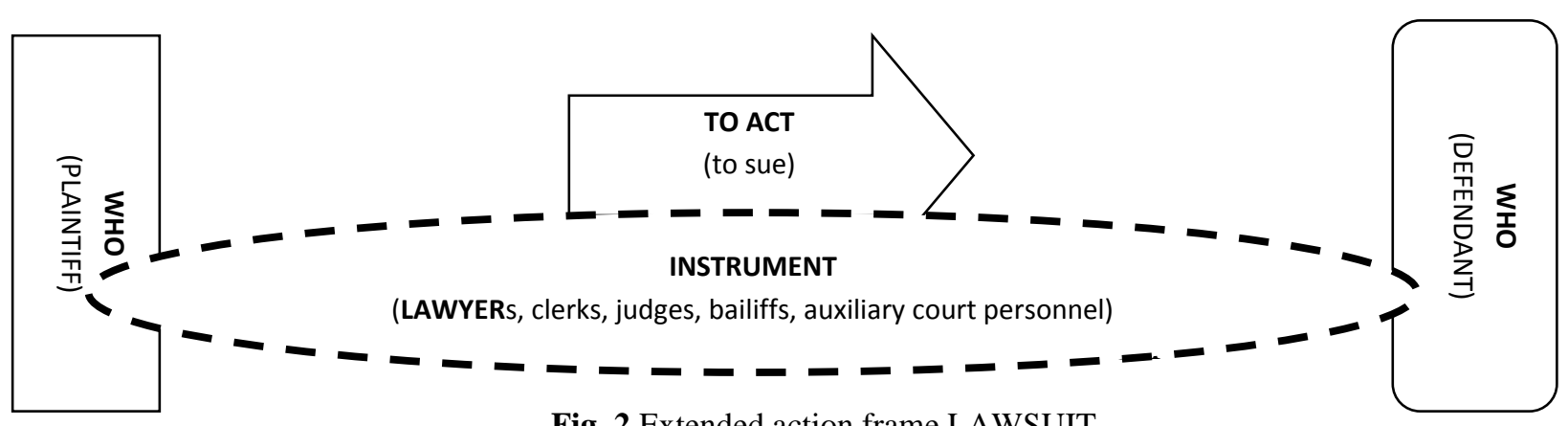

Fig. 2 Extended action frame LAWSUIT 
Figure 2 aims to depict the overlapping of the slots of the frame where the lexis from slot TO ACT interacts and as the result mixes with the implicated slot INSTRUMENT expending action frame LAWSUIT to: WHO (plaintiff) -- ACTS (sues) with an INSTRUMENT (lawyer/legal professional) -- WHO (defendant). The determined slots can serve as issue-related reservoirs for sorting the lexis corpus attributed to the study of particular field of law. Real Property Law [2, p. $52-53 ; 3$, p. 56-64] is chosen as an example, where lexis attributed to the lawsuit in the mentioned branch of law can be arranged in terms of action frame LAWSUIT in the following way:

$\checkmark \quad$ Slot WHO (plaintiff or defendant): landlord, landowner, tenant, heir, successor, spouse, surviving spouse, siblings, holder of property, landowner, real estate agent, property guardian etc.

$\checkmark \quad$ Slot TO ACT: to appoint, to receive, to file, to draft, to solve, to terminate, to restrict, to apply for, to appeal, to regulate, to convey, to defeat, to inherit etc.

$\checkmark \quad$ Slot INSTRUMENT: lawyers (solicitors, barristers, and advocates), clerks, judges, bailiffs, auxiliary court personnel, legal theorist etc.

The slots WHO (plaintiff) and (defendant) in terms of lexis can be contained within the same slot and where there is only the specified situation that will separate the roles between a defendant and a plaintiff, thus distributing the lexis between the slots performing the roles of specified situation participants. To some examples of specified situations in Real Property Law belong: the right of ownership, legal rights, personal property, property transactions, tenancy or leasehold estate, tangible property, intangible property, structures and minerals, interest in land, freehold estate, concurrent estate, incor- poreal interest, community property, undivided interest property etc.

Action frame approach can also be used in the case when for example, the data obtained from combined definition are assessed according to at least two different criteria for a situation to acquire the peculiarities of a notion where "Lawsuit" situation can serve as an example. This explains the differences in the variety of names of a process. Firstly, there is a lay definition of it as a case, problem, claim, dispute. Using a scheme of action frame a lawsuit is viewed as follows: WHO (person) -- ACTS (seeks a remedy from) -- WHO (person). Secondly, the same situation of "lawsuit" is named $a$ legal action, suit, proceeding at the very moment it enters the area of court of law. In terms of action frame it sounds in the following way: WHO (plaintiff) -ACTS (sues) -- WHO (defendant).

To sum it up, the research of action frame LAWSUIT and determination of the understanding a lawyer through it, offers a universal educational tool for arranging the knowledge and skills the students of law are acquiring in the classroom environment, since it addresses the real life situation of preparing and acting out a model of a lawsuit by a paralegal by means of English as foreign language. Furthermore, the example of frame analysis applied to a lawsuit situation enables the educators and learners to utilize a method in studying the various areas of law for example: Tort Law, Contract Law, Criminal Law, Real Property Law and many other. And what is more, this will help to develop the set of soft skills regarded as crucial for modern university education in law and gaining some deeper understanding of a professional environment through analysis of particular stereotypical situations.

\section{REFERENCES}

1. Bartlett F.C. Remembering: A Study in Experimental and Social Psychology, Cambridge University Press, 1967.

2. Wyatt R. Property, Check you English Vocabulary for Law, A\&C Black, London, 2006. P. $52-53$.

3. Callan H. and Edwards, L. Real Property Law, Absolute Legal English: for International Law, Delta Publishing, 2011. P. 56-64.

4. Fillmore Ch.J. Frame Semantics//Linguistics in the Morning Calm.Selected Papers from SICOL, Korea, Hunshin Publishing Company, Seoul, 1981. P. 110-137, available at: http://brenocon.com/Fillmore\%201982_2up.pdf (accessed 20th October 2019).

5. Fillmore Ch.J. Frames and the Semantics of Understanding//Quaderni di Semantica, 1985. II (6). P. 222-254, available http://www.icsi.berkeley.edu/pubs/ai/framesand85.pdf (accessed 20th October 2019).

6. Goodrich P. Legal Discourse. Studies in Linguistics, Rhetoric and Legal Analysis, Palgrave, Macmillan, UK, 1987.
7. Kuhn T. The Structure of Scientific Revolutions. University of Chicago Press, (2nd Ed.) 1970.

8. Minsky M. A Framework for Representing Knowledge. MIT-AI Laboratory Memo 306, 1974, available at: https://web.media.mit.edu/ minsky/papers/Frames/frames.h tml (accessed 02d December 2019).

9. Solam L. A., Ainsworth J., and Shuy R. W. Speaking of Language and Law (Edited by Lawrence M. Solan, Janet Ainsworth, and Roger W. Shuy), Oxford University Press, Oxford, UK, 2015.

10. Tiersma P. Legal English, University of Chicago Press, The USA, 1999.

11. Zhabotynska S.A. Principles of building conceptual models for thesaurus dictionaries//Cognition, communication, discourse. International On-line Journal, 2010. 1. P. 72-92, available http://sites.google.com/site/cognitiondiscourse/vypusk-no12010 (accessed 30 January 2019), DOI: 10.26565/22182926-2010-01-05

\footnotetext{
i https://www.ahdictionary.com

ii https://www.collinsdictionary.com

iii https://www.macmillandictionary.com

iv https://dictionary.cambridge.org

${ }^{v}$ http://dictionary.law.com

vi https://www.yourdictionary.com

vii https://www.lectlaw.com

https://www.uscourts.gov/glossary

viii http://www.businessdictionary.com
} 